International Mathematical Forum, 2, 2007, no. 67, 3339 - 3350

\title{
Higher-Order Difference and Higher-Order Splitting Methods for 2D Parabolic Problems with Mixed Derivatives
}

\author{
Jürgen Geiser \\ Department of Mathematics \\ Humboldt Universität zu Berlin \\ Unter den Linden 6, D-10099 Berlin, Germany \\ geiser@mathematik.hu-berlin.de
}

\begin{abstract}
In this article we discuss a combination between fourth-order finite difference methods and fourth-order splitting methods for $2 \mathrm{D}$ parabolic problems with mixed derivatives. The finite difference methods are based on higher-order spatial discretization methods, whereas the timediscretization methods are higher-order discretizations using CrankNicolson or BDF methods. The splitting methods are higher-order compact alternating direction implicit (ADI) methods. Here we construct a fourth-order splitting method with respect to the weighting factors. It is shown through a discrete Fourier analysis that the method is unconditionally stable in the diffusion case.
\end{abstract}

Mathematics Subject Classifications: 35J60, 35J65, 65M99, 65N12, 65Z05, 74S10, 76R50, 80A20, 80M25

Keywords: finite difference methods, 2D parabolic differential equations, operator-splitting methods, iterative methods, mixed derivatives

\section{Introduction}

Our motivation for this paper arose from different models in computational fluid dynamics. For such models it is essential to approximate the solution of the partial differential equation numerically in order to investigate the prediction of the models, as the analytical solutions are usually unavailable. We propose a splitting method for such approximations, that allow us to decompose the complicated multi-dimensional equation into simpler and applicable 
one-dimensional equations. For being as accurate as possible, the contribution of our research is to develop higher-order methods for the underlying splitting methods, that allow us to nearly skip the decomposition error. Traditionally using the finite difference and classical operator-splitting methods we obtain at least second-order methods. The simple decoupling of the differential equation into more basic equations, in which each equation contains only one operator, is often not sufficiently stable while the physical correlations between the operators are neglected.

From this basis on we are going to develop our methods using stable variants of finite difference schemes of fourth order, based on a weighted average factor $\mu$, considering the ADI principle. Further the modified ADI methods can be designed to be fourth-order accurate, while using the stabilization of the diffusion terms [14], [15]. We are going to examine the stability and consistency analysis based on the discrete Fourier analysis for these methods and adopt them to parabolic equations with mixed derivatives, see [17] and [21].

The paper is organized as follows. A mathematical model based on the wave equation is introduced in Section 2. The utilized discretization methods are described in Section 3. A modified ADI method for the parabolic equations is given in Section 4. The stability based on the discrete Fourier analysis is

discussed in Section 5. Finally we foresee our future works in the area of splitting and decomposition methods.

\section{Mathematical Model}

The motivation for the study presented below is coming from a computational simulation of fluid dynamics, see [12].

We concentrate on the scalar 2D parabolic equation [9], [15].

Let $\Omega$ be a rectangular domain in $\mathbb{R}^{2}$ with boundary $\Gamma=\partial \Omega$, and $J=(0, T]$ be the time interval with $T>0$. In our article, we consider the following equations:

$$
\begin{aligned}
& \partial_{t} u-\partial_{x x} u-\partial_{y y} u+\beta \partial_{x y} u+\alpha_{1} \partial_{x} u+\alpha_{2} \partial_{y} u=f, \text { in } \Omega \times J, \\
& u(x, y, 0)=u_{0}(x, y), \text { in } \Omega, \\
& u(x, y, t)=g(x, y, t), \text { on } \partial \Omega \times J \text { (Dirichlet boundary condition) } .
\end{aligned}
$$

The unknown function $u=u(x, y, t)$ is considered to be in $\Omega \times(0, T) \subset$ $\mathbb{R}^{2} \times \mathbb{R}$ and is the transport variable. The functions beta, $\alpha_{1}$, and $\alpha_{2}$ are smooth functions. Further $f, g$, and $u_{0}$ are sufficient smooth functions.

Equation (1) is applied in many models for convection-diffusion processes, concerning mass, heat, energy, temperature, etc. [14]. For example, the equation can be used for heat transfer in sublimation processes [9], chemical and 
radioactive waste transport [10] and transport of pollutants in the atmosphere [22].

For such problems, analytical solutions are not available for more complicated applications, therefore the benefit of numerical discretization and solver methods is important. In the following we discuss the approximation methods of higher order.

\section{Discretization Methods}

In the discretization methods, we apply for the spatial terms higher-order compact methods, which deal with finite difference methods [18].

\subsection{Spatial discretization (HOC)}

For the spatial discretization methods we apply higher-order compact methods (HOC), see [15].

For our Equation (1) without the mixed terms, the fourth-order discretization in space is given as

$$
L_{x} L_{y} u_{t}^{n}=\left(L_{y} A_{x}+L_{x} A_{y}\right) u^{n}+\mathcal{O}\left(h^{4}\right)
$$

with

$$
\begin{aligned}
& L_{x}=1+\frac{h_{x}^{2}}{12}\left(\delta_{x x}-\alpha_{1} \delta_{x}\right), \\
& A_{x}=-\left(1+\alpha_{1} \frac{h_{x}^{2}}{12}\right) \delta_{x x}+\alpha_{1} \delta_{x}, \\
& L_{y}=1+\frac{h_{y}^{2}}{12}\left(\delta_{y y}-\alpha_{2} \delta_{y}\right), \\
& A_{y}=-\left(1+\alpha_{2} \frac{h_{y}^{2}}{12}\right) \delta_{y y}+\alpha_{2} \delta_{y},
\end{aligned}
$$

where $h=\max \left\{h_{x}, h_{y}\right\} . \delta_{x}$ and $\delta_{y}$ are central difference operators for the first derivative, $\delta_{x x} u_{i}=\frac{u_{i+2}-u_{i}+u_{i-2}}{4 \Delta x^{2}}$ and $\delta_{y y}$ are the central difference operators for the second derivative.

The same idea can be used for the mixed term, then our operators are given 
as

$$
\begin{aligned}
& \tilde{L}_{x}=1+\frac{h_{x}^{2}}{12}\left(\delta_{x x}-\alpha_{1} \delta_{x}\right)+\frac{h_{x}^{2}}{12} \beta\left(\delta_{x x}+\delta_{x} \delta_{y}\right), \\
& \tilde{A}_{x}=-\left(1+\alpha_{1} \frac{h_{x}^{2}}{12}+\beta \frac{h_{x}^{2}}{12}\right) \delta_{x x}+\alpha_{1} \delta_{x}-\beta \delta_{x} \delta_{y}, \\
& \tilde{L}_{y}=1+\frac{h_{y}^{2}}{12}\left(\delta_{y y}-\alpha_{1} \delta_{y}\right)+\frac{h_{y}^{2}}{12} \beta \delta_{y y}, \\
& \tilde{A}_{y}=-\left(1+\alpha_{2} \frac{h_{y}^{2}}{12}+\beta \frac{h_{y}^{2}}{12}\right) \delta_{y y}+\alpha_{2} \delta_{y},
\end{aligned}
$$

where $h=h_{x}=h_{y}$, see also the formulation of the mixed discretization in [16].

We assume, that the operators $\tilde{L}_{x}$ and $\tilde{A}_{x}$ have the mixed derivative $\delta_{x} \delta_{y}$, where the other operators $\tilde{L}_{y}$ and $\tilde{A}_{y}$ are only depending on $y$. In such a case, we can also apply our decomposition method, and we call this a modified ADI method, which is not pure $x$ - and $y$-decomposing.

\subsection{Time-Discretization, CN and BDF3 Method}

For the time-discretization we apply the Crank-Nicolson method with an extrapolation to obtain a fourth-order method, as well as a backward differential formula method of third order (BDF3).

We now assume the semi-discretized equation, where we used a fourth-order spatial discretization method, as discussed in the previous subsection.

We discuss the semi-discretized equation given as

$$
\begin{aligned}
u_{t} & =f(u), \\
u(0) & =u_{0},
\end{aligned}
$$

where $f(u)$ can be assumed to be a linear or nonlinear operator, e.g. a matrix given from the spatial discretization.

Second order time-discretization method: Crank-Nicolson method We indicate the second-order Crank-Nicolson method, which is given as

$$
\begin{gathered}
\frac{u^{n+1}-u^{n}}{\Delta t}=1 / 2\left(f\left(u^{n}\right)+f\left(u^{n+1}\right)\right), \\
u(0)=u_{0},
\end{gathered}
$$

where $u^{n}$ is the time-approximated solution at $t^{n}=n \Delta t, n \geq 0 . \Delta t$ denotes the time increment.

The scheme is given as

$$
\begin{gathered}
S_{C N}\left(\Delta t, u^{n}\right)=u^{n+1, C N}=u^{n}+\frac{\Delta t}{2}\left(f\left(u^{n}\right)+f\left(u^{n+1}\right)\right), \\
S_{C N}\left(\Delta t, u^{n}\right)=B\left(\frac{\Delta t}{2}, F\left(\frac{\Delta t}{2}, u^{n}\right)\right),
\end{gathered}
$$


where

$$
\begin{gathered}
B\left(\frac{\Delta t}{2}, u^{n+1 / 2}\right)=u^{n+1, B}=u^{n+2}+\frac{\Delta t}{2} f\left(u^{n+1}\right) \\
F\left(\frac{\Delta t}{2}, u^{n}\right)=u^{n+1 / 2, F}=u^{n}+\frac{\Delta t}{2} f\left(u^{n}\right) .
\end{gathered}
$$

Based on this second-order method, we can apply an extrapolation method to obtain a higher-order method, which we need for our modified ADI method.

\section{Higher-order method based on Richardson extrapolation}

We apply the Richardson extrapolation on the second-order Crank-Nicolson method to obtain higher-order methods.

The idea of the extrapolation method is given as follows.

$$
D_{4}\left(\Delta t, u^{n}\right)=u^{n+1,4 t h}=\frac{4}{3} S_{C N}\left(\frac{\Delta t}{2}, S_{C N}\left(\frac{\Delta t}{2}, u^{n}\right)\right)-\frac{1}{3} S_{C N}\left(\Delta t, u^{n}\right) .
$$

To obtain fifth order, we have to apply a Richardson extrapolation additionally, see [2], [19]:

$$
D_{5}\left(\Delta t, u^{n}\right)=u^{n+1,5 t h}=\frac{16}{15} D_{4}\left(\frac{\Delta t}{2}, D_{4}\left(\frac{\Delta t}{2}, u^{n}\right)\right)-\frac{1}{15} D_{4}\left(\Delta t, u^{n}\right) .
$$

These methods can be implemented with respect to the basic time-discretization method. Another method, which we can obtain a higher order, is given in the following part.

\section{SBDF3 Method}

We use the following stiff backward differential formula (SBDF) method, which is a modification of the third-order backward differential formula (BDF3).

We devide the operator $f(u)$ in $A(u)=f(u) / 2$ and $B(u)=f(u) / 2$, see [1] and $[11]$.

$$
\begin{aligned}
& \frac{1}{\Delta t}\left(\frac{11}{6} u^{n+1}-3 u^{n}+\frac{3}{2} u^{n-1}-\frac{1}{3} u^{n-2}\right) \\
& =\frac{3}{2} f\left(u^{n}\right)-\frac{3}{2} f\left(u^{n-1}\right)+\frac{1}{2} f\left(u^{n-2}\right)+\frac{1}{2} f\left(u^{n+1}\right),
\end{aligned}
$$

where $u^{n}=u\left(t^{n}\right)$ is the solution given at time $t^{n}$. Further $f(u)$ is a sufficient smooth function.

For this explicit-implicit scheme, we can construct an ADI method of third order. The decoupling for the ADI method is obtained because of the implicitexplicit character of the method.

In the next section we discuss the higher-order ADI methods for the diffusion equation. 


\section{Modified ADI methods}

Our method is based on the classical ADI method of [4] and [15], which decouples into the different spatial terms of the equations.

In a first method we apply the pure diffusion case and obtain for such an equation a stable higher-order splitting method.

\subsection{3rd-order ADI scheme}

We apply the ideas to construct an ADI method as given in [4] and [15].

A third-order ADI method is given in the following proposition.

Proposition 4.1 The ADI method based on the SBDF3 method is given as

$$
\begin{aligned}
& \left(L_{x}+\alpha \Delta t A_{x}\right) u^{* * *}=-\Delta t L^{*} u^{* *}-\Delta^{2} t / 2 A_{x} A_{y} u^{* *}, \\
& \left(L_{y}+\alpha \Delta t A_{y}\right) u^{*}=u^{* * *},
\end{aligned}
$$

where $L^{*}=L_{y} A_{x}+L_{x} A_{y}$ and $u^{*}=\left(\frac{11}{6} u^{n+1}-3 u^{n}+\frac{3}{2} u^{n-1}-\frac{1}{3} u^{n-2}\right)$,

$$
u^{* *}=\beta u^{n}+\gamma u^{n-1}+\delta u^{n-2} \text {. }
$$

The coefficients are given as

$$
\begin{aligned}
\alpha & =\frac{1}{2} \frac{6}{11}=\frac{3}{11} \\
\beta & =\frac{3}{2}-3 \alpha=\frac{15}{22} \\
\gamma & =-\frac{3}{2}+\frac{3}{2} \alpha=-\frac{12}{11} \\
\delta & =\frac{1}{2}-\frac{1}{2} \alpha=\frac{4}{11}
\end{aligned}
$$

Proof. For the third-order ADI method we construct the method based on the following idea of the third-order SBDF method:

$$
\begin{aligned}
& L_{x} L_{y}\left(\frac{11}{6} u^{n+1}-3 u^{n}+\frac{3}{2} u^{n-1}-\frac{1}{3} u^{n-2}\right) \\
& =\Delta t L^{*}\left(\frac{3}{2} u^{n}-\frac{3}{2} u^{n-1}+\frac{1}{2} u^{n-2}+\frac{1}{2} u^{n+1}\right),
\end{aligned}
$$

where $L^{*}=L_{y} A_{x}+L_{x} A_{y}$.

The Equation (10) is equivalent to the following Equation (11), that results of the construction.

$$
\begin{aligned}
& \left(L_{x} L_{y}+\alpha \Delta t L^{*}\right)\left(\frac{11}{6} u^{n+1}-3 u^{n}+\frac{3}{2} u^{n-1}-\frac{1}{3} u^{n-2}\right) \\
& =\Delta t L^{*}\left(\beta u^{n}+\gamma u^{n-1}+\delta u^{n-2}\right)+\mathcal{O}\left(\Delta t^{4}\right)
\end{aligned}
$$


where $L^{*}=L_{y} A_{x}+L_{x} A_{y}, u^{*}=\left(\frac{11}{6} u^{n+1}-3 u^{n}+\frac{3}{2} u^{n-1}-\frac{1}{3} u^{n-2}\right)$.

We can derive the coeffients by comparing Equations (10) and (11).

As a result we obtain the factors $\alpha, \beta, \gamma$ and $\delta$ as given in the proposition.

Then the ADI splitting method is given as:

$$
\begin{aligned}
& \left(L_{x}+\alpha \Delta t A_{x}\right) u^{* * *}=-\Delta t L^{*} u^{* *}-\Delta^{2} t / 2 A_{x} A_{y} u^{* *}, \\
& \left(L_{y}+\alpha \Delta t A_{y}\right) u^{*}=u^{* * *}
\end{aligned}
$$

where $L^{*}=L_{y} A_{x}+L_{x} A_{y}, u^{*}=\left(\frac{11}{6} u^{n+1}-3 u^{n}+\frac{3}{2} u^{n-1}-\frac{1}{3} u^{n-2}\right)$, and $u^{* *}=$ $\beta u^{n}+\gamma u^{n-1}+\delta u^{n-2}$.

\subsection{Fourth-order ADI scheme}

To obtain a fourth-order ADI method, the underlying time-discretization method has to be at least fourth-order in time.

So we support our new method with the Richard extrapolation that uses the second-order ADI method, based on the Crank-Nicolson time discretisation, and we reach at least a fourth-order method.

For the second-order ADI method we apply the second-order time-discretization given as Crank-Nicolson (CN) method.

The $\mathrm{CN}$ time discretisation is given as

$$
\left(L_{x} L_{y}+\frac{\Delta t}{2} L^{*}\right) u^{n+1}=\left(L_{x} L_{y}-\frac{\Delta t}{2} L^{*}\right) u^{n}+O\left(h^{4}\right)+O\left(\Delta t^{3}\right),
$$

where the discretisation is of order 2 in time and order 4 in space.

The ADI-method, following [3], is given as follows.

$$
\begin{aligned}
& \left(L_{x}+\frac{\Delta t}{2} A_{x}\right) u^{*}=\left(L_{x}-\frac{\Delta t}{2} A_{x}\right)\left(L_{y}-\frac{\Delta t}{2} A_{y}\right) u^{n}, \\
& \left(L_{y}+\frac{\Delta t}{2} A_{y}\right) u^{n+1}=u^{*},
\end{aligned}
$$

where we obtain a second order ADI-scheme.

By appling the Richardson extrapolation we obtain a fourth-order method for the CN scheme, see scheme (5).

Proposition 4.2 The ADI-method based on the extrapolation and CN method is given in the next steps.

Step 1 (first $\Delta t / 2$ step), $\alpha=1 / 2$ :

$$
\begin{aligned}
& \left(L_{x}+\alpha \frac{\Delta t}{2} A_{x}\right) u^{*, n+1 / 2}=\left(L_{x}-\alpha \frac{\Delta t}{2} A_{x}\right)\left(L_{y}-\alpha \frac{\Delta t}{2} A_{y}\right) u^{n}, \\
& \left(L_{y}+\alpha \frac{\Delta t}{2} A_{y}\right) u^{n+1 / 2}=u^{*, n+1 / 2},
\end{aligned}
$$


Step 2 ( $\Delta t$ step), $\alpha=1.0$ :

$$
\begin{aligned}
& \left(L_{x}+\alpha \frac{\Delta t}{2} A_{x}\right) u^{*, n+1}=\left(L_{x}-\alpha \frac{\Delta t}{2} A_{x}\right)\left(L_{y}-\alpha \frac{\Delta t}{2} A_{y}\right) u^{n}, \\
& \left(L_{y}+\alpha \frac{\Delta t}{2} A_{y}\right) \tilde{u}^{n+1}=u^{*, n+1},
\end{aligned}
$$

Step 3 (second $\Delta t / 2$ step), $\alpha=1 / 2$ :

$$
\begin{aligned}
& \left(L_{x}+\alpha \frac{\Delta t}{2} A_{x}\right) u^{*, n+1}=\left(L_{x}-\alpha \frac{\Delta t}{2} A_{x}\right)\left(L_{y}-\alpha \frac{\Delta t}{2} A_{y}\right) u^{n+1 / 2}, \\
& \left(L_{y}+\alpha \frac{\Delta t}{2} A_{y}\right) \tilde{\tilde{u}}^{n+1}=u^{*, n+1}
\end{aligned}
$$

Resulting step:

$$
u^{n+1}=4 / 3 \tilde{\tilde{u}}^{n+1}-1 / 3 \tilde{u}^{n+1}
$$

where we obtain a 4th order method, due to the Richardson Extrapotation with 2nd order methods.

Proof. By including the 3 substeps of the Richardson extrapolation (5) to the ADI method, we can derive the result of Proposition 4.2.

\section{$5 \quad$ Stability Analysis}

In this section we proove the stability of the ADI method based on SBDF3 methods. The underlying stability proof is based on the linear multistep methods and we apply the discrete Fourier transform [13].

steps.

Our underlying multistep method is given as:

$$
\alpha_{k} y_{n+k}+\alpha_{k-1} y_{n+k-1}+\ldots \alpha_{0} y_{n}
$$

We have the following general solutions.

Lemma 5.1 Let $\zeta_{1}, \zeta_{2}, \ldots, \zeta_{l}$ be roots of $\rho(\zeta)$ with respective multiplicities $m_{1}, \ldots, m_{l}$. Then the general solution of (22) is given by

$$
y_{n}=p_{1}(n) \zeta_{1}^{n}+\ldots+p_{l}(n) \zeta_{l}^{n},
$$

where the $p_{j}(n)$ are polynomials of degree $m_{j}-1$.

We define a stable multistep method as follows.

Definition 5.2 The multistep method (22) is called stable, if the generating polynomial $\rho(\zeta)$ satisfies the root condition, i.e. 
- the roots of $\rho(\zeta)$ lie on or within the unit circle,

- the roots on the unit circle are simple.

To study the stability of the scheme, we can use the Neumann linear stability analysis and proof the following theorem.

Theorem 5.3 The ADI method, given in Equation (8) and (9) and based on the SBDF3 method, is unconditionally stable.

Proof. After the Fourier transform of Equations (8)-(9), we obtain

$$
\begin{aligned}
& \left(\gamma_{1}^{x}+2 \alpha \gamma_{2}^{x}\right) \bar{u}^{* * *}=-2\left(\gamma_{1}^{x} \gamma_{2}^{x}+\gamma_{2}^{x} \gamma_{1}^{y}\right) \bar{u}^{* *} \\
& \left(\gamma_{1}^{y}+\alpha \gamma_{2}^{y}\right) \bar{u}^{*}=\bar{u}^{* * *}
\end{aligned}
$$

where

$$
\begin{aligned}
& \bar{u}^{*}=\frac{11}{6} \zeta^{3}-3 \zeta^{2}+\frac{3}{2} \zeta-\frac{1}{3}, \\
& \bar{u}^{* *}=\beta \zeta^{2}+\gamma \zeta+\delta .
\end{aligned}
$$

The resulting polynom for the stability is given as

$$
\begin{aligned}
& \left.\zeta^{3}+\zeta^{2} \gamma_{1}^{x}+2 \alpha \gamma_{2}^{x}\right) \bar{u}^{* * *}=-2\left(\gamma_{1}^{x} \gamma_{2}^{y}+\gamma_{2}^{x} \gamma_{1}^{y}\right) \bar{u}^{* *} \\
& \left(\gamma_{1}^{y}+\alpha \gamma_{2}^{y}\right) \bar{u}^{*}=\bar{u}^{* * *}
\end{aligned}
$$

where the coeffients are

$$
\begin{aligned}
\gamma_{1}^{x} & =1-1 / 3 \sin ^{2}\left(\theta_{x} / 2\right), \\
\gamma_{2}^{x} & =2 \frac{\Delta t}{h^{2}} \sin ^{2}\left(\theta_{x} / 2\right),
\end{aligned}
$$

with $\theta_{x}$ being the phase angle in direction $x$. A similar definition can be used for the terms $\gamma_{1}^{y}$ and $\gamma_{2}^{y}$.

We obtain the following polynomial

$$
\zeta^{3}+f_{1}\left(\gamma_{1}^{x}, \gamma_{1}^{y}, \gamma_{2}^{x}, \gamma_{2}^{y}\right) \zeta^{2}+f_{2}\left(\gamma_{1}^{x}, \gamma_{1}^{y}, \gamma_{2}^{x}, \gamma_{2}^{y}\right) \zeta+f_{3}\left(\gamma_{1}^{x}, \gamma_{1}^{y}, \gamma_{2}^{x}, \gamma_{2}^{y}\right)=0(28)
$$

where the coefficients are

$$
\begin{aligned}
& f_{1}=\frac{\alpha_{11} \gamma_{1}^{x}+\alpha_{12} \gamma_{1}^{y}+\alpha_{13} \gamma_{2}^{x}+\alpha_{14} \gamma_{2}^{y}}{\alpha_{1} \gamma_{1}^{x}+\alpha_{2} \gamma_{1}^{y}+\alpha_{3} \gamma_{2}^{x}+\alpha_{4} \gamma_{2}^{y}}, \\
& f_{2}=\frac{\alpha_{21} \gamma_{1}^{x}+\alpha_{22} \gamma_{1}^{y}+\alpha_{23} \gamma_{2}^{x}+\alpha_{24} \gamma_{2}^{y}}{\alpha_{1} \gamma_{1}^{x}+\alpha_{2} \gamma_{1}^{y}+\alpha_{3} \gamma_{2}^{x}+\alpha_{4} \gamma_{2}^{y}}, \\
& f_{3}=\frac{\alpha_{31} \gamma_{1}^{x}+\alpha_{32} \gamma_{1}^{y}+\alpha_{33} \gamma_{2}^{x}+\alpha_{34} \gamma_{2}^{y}}{\alpha_{1} \gamma_{1}^{x}+\alpha_{2} \gamma_{1}^{y}+\alpha_{3} \gamma_{2}^{x}+\alpha_{4} \gamma_{2}^{y}}, \\
& f_{4}=\frac{\alpha_{41} \gamma_{1}^{x}+\alpha_{42} \gamma_{1}^{y}+\alpha_{43} \gamma_{2}^{x}+\alpha_{44} \gamma_{2}^{y}}{\alpha_{1} \gamma_{1}^{x}+\alpha_{2} \gamma_{1}^{y}+\alpha_{3} \gamma_{2}^{x}+\alpha_{4} \gamma_{2}^{y}}
\end{aligned}
$$


The coefficients can be computed by Matlab or Mathematica and we have the following results.

$$
\begin{aligned}
& \max \left(\alpha_{11}, \alpha_{21}, \alpha_{31}, \alpha_{41}\right) \leq \alpha_{1} \\
& \max \left(\alpha_{12}, \alpha_{22}, \alpha_{32}, \alpha_{42}\right) \leq \alpha_{2}, \\
& \max \left(\alpha_{13}, \alpha_{23}, \alpha_{33}, \alpha_{43}\right) \leq \alpha_{3}, \\
& \max \left(\alpha_{14}, \alpha_{24}, \alpha_{34}, \alpha_{44}\right) \leq \alpha_{4}
\end{aligned}
$$

For all functions holds $\left|f_{i}\right| \leq 1$, and therefore the roots are in the unit circle, see $[13]$.

Therefore the method is unconditionally stable.

Remark 5.4 Because of the pure diffusion case we could derive the unconditionally stable results. As a weaker formulation, the influence of the convection or mixed term may destabilize the method. We assume for our further experiments sufficient stability, while applying sufficient small time steps.

\section{Conclusions and Discussions}

We present modified ADI methods, which can be derived as 3rd and 4th order methods, based on the higher time-discretizations. We discuss the stability analysis for the pure diffusive case and derive unconditionally stability. Based on this result, we apply the general equation in several experiments to validate the applicability to complicated models. As a result, the higher-order ADI methods benefit in saving computational time to obtain higher accurate solutions with less amount. In future the stability analysis will be generalized to mixed derivatives.

\section{References}

[1] U.M. Ascher, St.J. Ruuth, and B.T.R. Wetton. Implicit-Explicit Methods for Time-Dependent Partial Differential Equations. SIAM Journal on Numerical Analysis, Vol. 32, No. 3, pp. 797-823, 1995.

[2] St. Descombes. Convergence of a splitting method of high order for reaction-diffusion systems. Mathematics of Computations, 70, 1481-1501, 2001.

[3] E.G. D'yakonov. Difference schemes of second order accuracy with a splitting operator for parabolic equations without mixed derivatives. Zh. Vychisl. Mat. I Mat. Fiz.(in Russian), vol. 4, 935-941, 1964. 
[4] J. Douglas Jr. and J.E. Gunn. A general formulation of alternating direction methods. I. Parabolic and hyperbolic problems. Numerische Mathematik, 6, 428-453, 1964.

[5] K.-J. Engel, R. Nagel, One-Parameter Semigroups for Linear Evolution Equations. Springer, New York, 2000.

[6] I. Farago, J. Geiser. Iterative Operator-Splitting methods for Linear Problems. Preprint No. 1043 of the Weierstrass Institute for Applied Analysis and Stochastics, Berlin, Germany, June 2005.

[7] J. Geiser. Discretization Methods with embedded analytical solutions for convection dominated transport in porous media Proceeding of Numerical Analysis and Applications, Third international conference, Rousse, Bulgaria, 2004, Lect.Notes in Mathematics (Springer), vol.3401, 2005.

[8] J. Geiser, R.E. Ewing, J. Liu. Operator Splitting Methods for Transport Equations with Nonlinear Reactions. Proceedings of the Third MIT Conference on Computational Fluid and Solid Mechanic, Cambridge, MA, June 14-17, 2005.

[9] J. Geiser. Discretization methods with analytical solutions for convectiondiffusion-dispersion-reaction-equations and application. Journal of Engineering Mathematics, Springer Verlag, Berlin, Heidelberg, 57, 79-98, 2007.

[10] J. Geiser. A Numerical Investigation for a Model of the Solid-Gas Phase in a Crystal Growth Apparatus. Communications in Computational Physics (CiCP), Global Science Press, accepted, September 2007.

[11] J. Geiser, Joscha Gedicke. Iterative Operator-Splitting Methods with higher order Time-Integration Methods and Applications for Parabolic Partial Differential Equations. Humboldt-Preprint, No. 2006-10, March 2006.

[12] R. Glowinski. Numerical methods for fluids. Handbook of Numerical Analysis, Gen. eds. P.G. Ciarlet, J. Lions, Vol. IX, North-Holland Elsevier, Amsterdam, The Netherlands, 2003.

[13] E. Hairer, S.P. Norsett, and G. Wanner. Solving Ordinary Differential Equatons I. SCM, Springer-Verlag Berlin-Heidelberg-New York, No. 8, 1992.

[14] W. Hundsdorfer and J.G. Verwer. Numerical Solution of Time-dependent Advection-Diffusion-Reaction Equations. Springer Series in Computational Mathematics, 33, Springer Verlag, 2003. 
[15] S. Karaa. High-Order Compact ADI Methods for Parabolic Equations. Journal of Computers and Mathematics with Applications, Vol. 52, Iss. 8-9, 1343-1356, 2006.

[16] S. Karaa. High-Order Difference Schemes for 2-d Elliptic and Parabolic Problems with Mixed Derivatives. Wiley InterSciences, published online, October 2006.

[17] R.J. Leveque. Finite Difference Methods for Ordinary and Partial Differential Equations. OT98, SIAM, Philadelphia, USA, 2007.

[18] B.J. Noye and H.H. Tan. Finite difference methods for solving the two dimensional advection-diffusion equation. Int. J. Numer. Methods Fluids, 26, 1615-1629, 1988.

[19] J. Stoer and R. Burlisch. Introduction to Numerical Analysis. 3rd. ed. , Springer-Verlag, New-York, 2002.

[20] G. Strang. On the construction and comparision of difference schemes. SIAM J. Numer. Anal., 5:506-517, 1968.

[21] J.C. Strikwerda. Difference Schemes and Partial Differential Equations. Wadsworth and Brooks-Cole, Pacific Grove, CA, 1989.

[22] Z. Zlatev. Computer Treatment of Large Air Pollution Models. Kluwer Academic Publishers, 1995.

Received: September 28, 2007 